\title{
Adaptive Fuzzy Control for Aircraft Tractor's Magneto Rheological Semi-active Suspension
}

\author{
Li Jun Zhao ${ }^{1, a}$, Xiao Cheng ${ }^{1, b}$ and Qing Fu Wei ${ }^{1, c}$ \\ ${ }^{1}$ Harbin Institute of Technology, Weihai, 264209, China \\ azhaolijun@hitwh.edu.cn, b15069426785@163.com, 'weiqingfu@163.com
}

\begin{abstract}
Keywords: Aircraft tractor; Semi-active suspension; Magneto rheological damper; Adaptive fuzzy control; Adjustable factor

Abstract. In order to improve aircraft tractor's ride comfort and traction friendly, this paper takes the MR semi-active suspension as the research object and establishes half body model. Through fitting, this paper obtained the magnetic rheological damper's damping expression, designed a magnetic rheological damper with low pressure nitrogen compensation structure. The fuzzy control is used to study suspension's performance. Aiming for reducing body acceleration, this paper introduced the thought of adaptive control to adjust the domain constantly. The simulation results shows that the adaptive fuzzy control can improve aircraft tractor's suspension function obviously with small amount of calculation, good real-time performance. This algorithm has self-adaptability to the changes in the suspension parameters, easy to meet the control system's requirement and realize in engineering.
\end{abstract}

\section{Introduction}

Aircraft tractor is an important airport equipment which works at the airport by pulling or pushing to achieve short-distance aircraft movement[1]. Recent years, with advances in materials science and breakthrough in magneto-rheological anti-settling technology, magneto-rheological damper has been widely used in various fields because of its precise real-time control, capable of continuous change and reversible damping force, simple mechanical and electrical construction and energy less consumption[2].Suspension is a complex, nonlinear systems. Since classical control theory is not easy to control, there already have many studies using modern control theory and intelligent control theory. [3] used optimal control algorithm for quarter body model. The results showed that control is better than passive suspension, but the choice of weighting coefficients mainly depends on the designer's experience, lacking theoretical basis; [4] used robust control, however not only computing increase but controller is conservative and real-time is bad; Fuzzy control does not depend on the precise mathematical model and has advantages in dealing with non-linear problems. With characteristic of self-tuning adaptive control, it can get very good control effect [5].

\section{Semi-active Suspension System for Aircraft Tractors}

Mathematical Model of Suspension System. Aircraft tractor's front wheels adopt independent suspension and rear wheels were directly connected with the body. Half body model is appropriate. A simplified model of the vehicle body is shown in Figure 1.

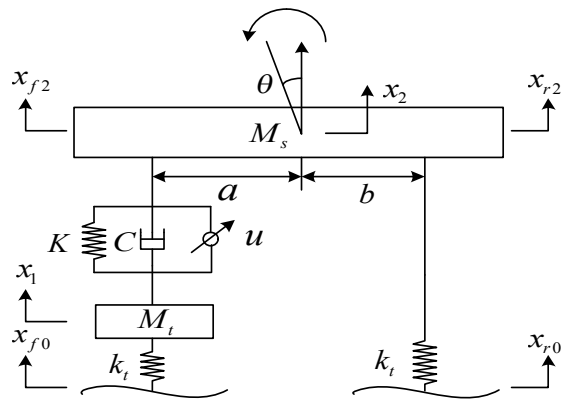

Fig. 1 Half body model of aircraft tractor

When the aircraft tractor pitch angle is relatively small, relationship between front wheel sprung 
mass displacement and the upper body displacement and the aircraft wheel tractor centroid displacement can be expressed with equation (1):

$$
\left\{\begin{array}{l}
x_{f 2}=x_{2}-a \tan \theta=x_{2}-a \theta \\
x_{r 2}=x_{2}+b \tan \theta=x_{2}+b \theta
\end{array}\right.
$$

Mathematical Model of Magneto-rheological Damper. The structure of designed magnetorheological damper based on mixed mode with low pressure nitrogen compensation device is shown in Figure 2.

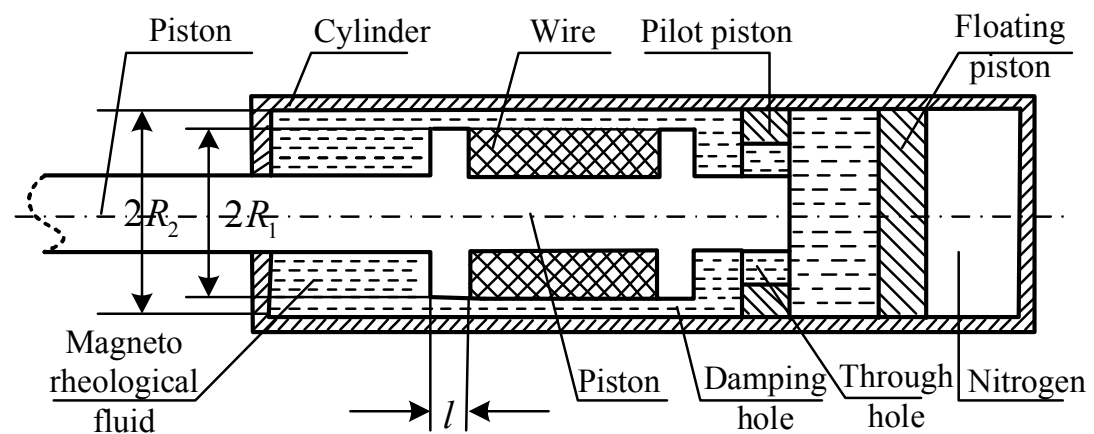

Fig.2 Magnetic rheological damper structure diagram

Currently, the establishment of magneto-rheological damper mainly relies on the method of mechanical model tests. [6] and [7] pointed magneto-rheological shock absorber damping force can be expressed in equation(2) :

$$
F=\frac{12 \eta l A_{p}^{2}}{\pi R_{1}\left(R_{2}-R_{1}\right)^{3}} v+\frac{6 l A_{p} K}{R_{2}-R_{1}}\left[\frac{N I}{2\left(R_{2}-R_{1}\right)}\right]^{\beta} \operatorname{sgn}(v)
$$

In in equation, $\eta$ is MRF zero-field viscosity; $A_{p}$ is the effective area of the piston; $K$ and $\beta$ is MRF test constants; $N$ is the number of turns; $I$ is the intensity of the electric current.

After checking, when $l=0.01 \mathrm{~m}, R_{1}=0.0305 \mathrm{~m}, N=245$, the damping force - velocity curve of magneto-rheological shock absorber is shown in Figure 3. So the control current can be obtained according to equation (1) after getting adaptive fuzzy control of damping force.

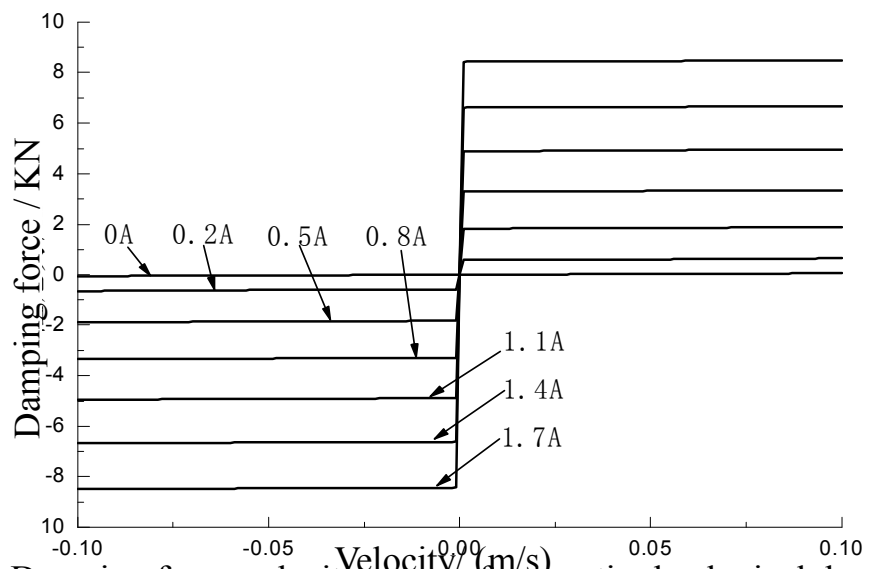

Fig.3.Damping force-velocity curve of mâgnetic rheological damper

\section{Adaptive Fuzzy Control System Structure}

Structure design of adaptive fuzzy controller. This article takes acceleration error and derivative of sprung mass as the input of adaptive fuzzy control system. So it is a two-inputs and single-output system which structure is shown in Figure 4. Since the inputs and output are functions of time whose domains can be written as $X(x(t)), Y(y(t))$ and $Z(u(t))$. Fuzzy control rule is: 
If $x(t)$ is $\tilde{A}_{i}(t)$ and $y(t)$ is $\tilde{B}_{j}(t)$ then $u(t)$ is $\tilde{C}_{i j}(t)$ 。

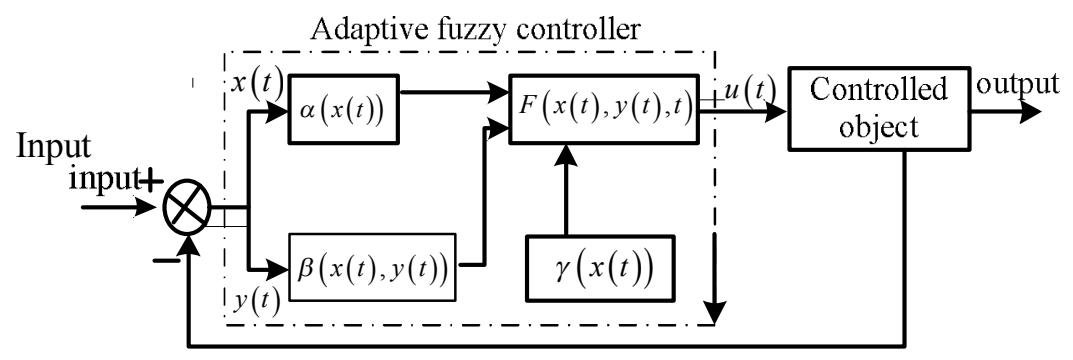

Fig.4 Adaptive fuzzy control structure of two input and one output

Determine of Domain and Quantitative Factors. In order to simplify the set of control parameters, usually fuzzy domain was set integer. This process is called variable discrete. Assume three fuzzy subsets domains are:

$$
\begin{gathered}
\{-n,-n+1,-n+2, \cdots, n-2, n-1, n\} \\
\{-m,-m+1,-m+2, \cdots, m-2, m-1, m\} \\
\{-l,-l+1,-l+2, \cdots, l-2, l-1, l\} \\
n, m, l=6
\end{gathered}
$$

Quantization factor refers to the selected scale factor when transforming the accurate physical variables into fuzzy variables. When the aircraft tractor drives at speed of $5 \mathrm{~m} / \mathrm{s}$ on an A level road, conversion factors of the inputs and output are determined by simulations, i.e. $k_{e}=4.2, k_{e c}=0.14, k_{u}=500$ respectively.

Determine of Adaptive Stretching Factor. Define $\alpha: X \rightarrow[0,1], x \mid \rightarrow \alpha(x)$ as stretching factor of domain, i.e. $X$.Sprung mass acceleration and its derivatives are related, should consider the synergy between them, stretching factor is expressed as:

$$
\alpha(x, y)=1-\lambda_{x, y} e^{\left(-k_{x, y} x^{2}-k_{y, x} y^{2}\right)}
$$

In which, $\lambda_{x, y}$ is the scaling factor of expansion factor for bivariate input, $0<\lambda_{x, y}<1 . k_{x, y}$ and $k_{y, x}$ are the index factors of expansion factor for bivariate input, $0<k_{x, y}<1,0<k_{y, x}<1$.

Control Algorithm for Adaptive Fuzzy. Essence of fuzzy control algorithm is a kind of interpolation function [8]. Adaptive fuzzy control is no exception which can be written as follow:

$$
y(t)=\beta(t) U \sum_{j=1}^{m} \prod_{i=1}^{n} A_{i j}\left(\frac{x_{i}(t)}{\alpha_{i}\left(x_{i}(t)\right)}\right) y_{j}
$$

This article applied a new point of view. Assuming theoretical domain of input is $X=[-x, x]$. Through adaptive fuzzy control, the theoretical domain (generally become smaller) is bound to change. We could first make the input variables time the inverse of scaling factor, then applying it into the case of the adaptive control system, so we can keep the domain of body and control rules of fuzzy control does not change without reducing the accuracy of fuzzy control [9].The structure is shown in Figure 5.

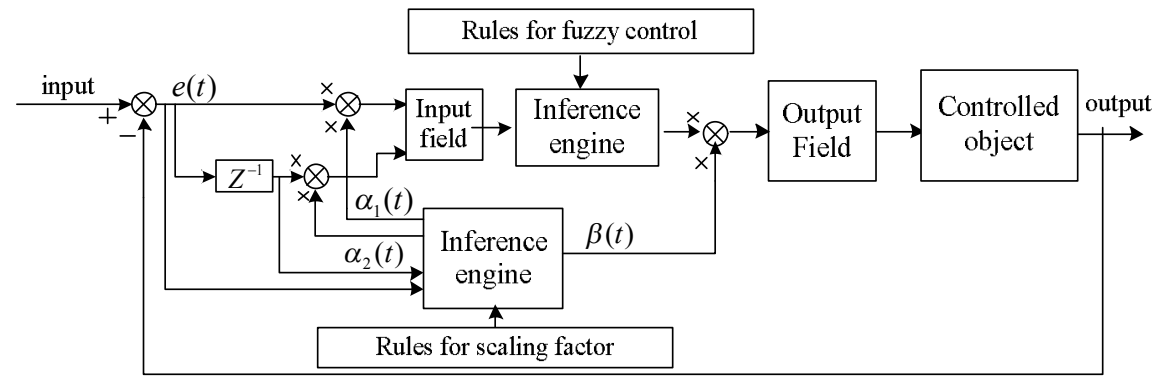

Fig.5 Principle diagram of adaptive fuzzy control 


\section{Building Road Input Model}

As the vehicle vibration input, road roughness mainly adopt road power spectral density describing its statistical properties. The expression of velocity power spectral density is $G_{\dot{q}}(n)=\left(2 \pi n_{0}\right)^{2} G_{q}\left(n_{0}\right)$. In which, $n$ is the spatial frequency; $n_{0}$ is reference spatial frequency; $G_{q}\left(n_{0}\right)$ is road power spectral density at frequency value of $n_{0}$. It is a constant at the entire frequency range, i.e. "white noise." In order to facilitate analysis of the problem in the state space, converted it into a form of time domain:

$$
G_{\dot{q}}(f)=(2 \pi f)^{2} G_{q}(f)=4 \pi^{2} G_{q}\left(n_{0}\right) n_{0}^{2} v
$$

In which, $v$ is the speed of vehicle. Random road contours was set up through equation (4).

\section{The Simulation Results Analysis}

According to the above half body model, adaptive fuzzy control algorithm and road model, this article established a simulation model in Simulink. Assuming the aircraft tractor with a speed of $5 \mathrm{~m} / \mathrm{s}$, the simulation results are shown in Fig. 6 to 9.Performance indicators for passive suspension and adaptive fuzzy control are showed in Table 1.

As can be seen, for semi-active suspension using magneto-rheological adaptive fuzzy control, body acceleration and suspension displacement have significantly reduced, indicating a better performance than passive suspension. The deformation of the tire is no better than that of passive suspension. This is mainly because the fuzzy control rules are developed out of the purpose of reducing the body acceleration which has almost no effects on the low-speed vehicles such aircraft tractor.

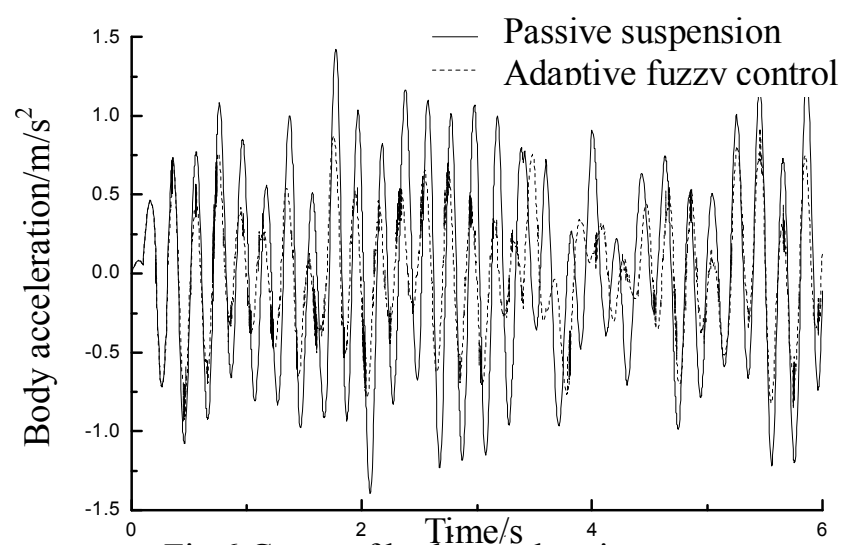

Fig. 6 Curve of body acceleration

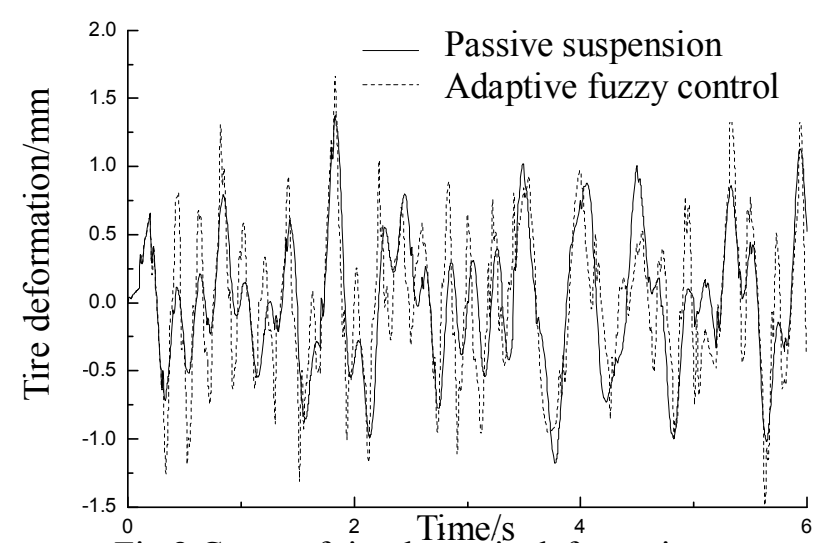

Fig.8 Curve of tire dynamic deformation

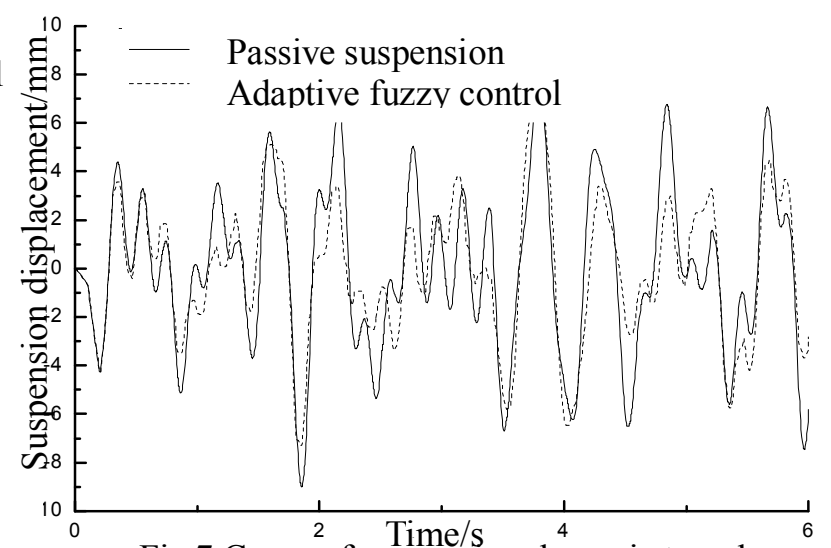

Fig.7 Curve of suspension dynamic travel

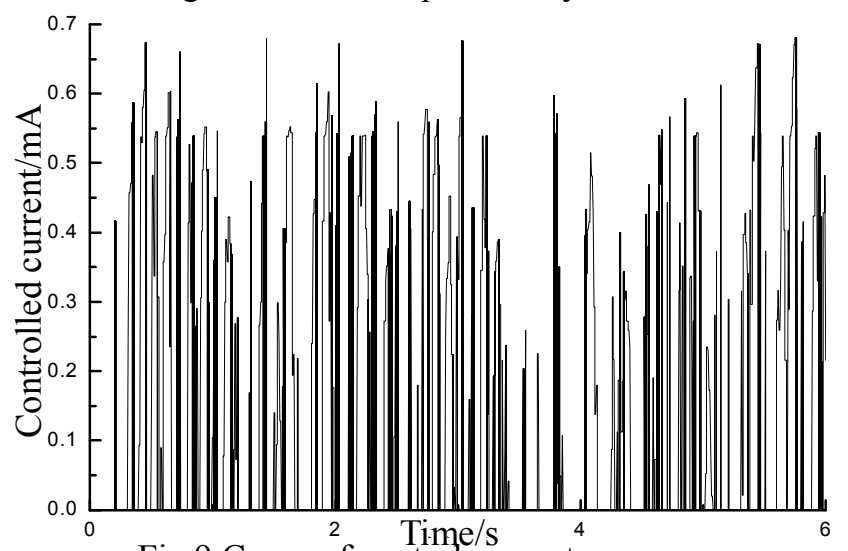

Fig. 9 Curve of control current 
Table.1. Performance index of passive suspension and adaptive fuzzy control suspension

\begin{tabular}{lccc}
\hline Performance index & Passive suspension & $\begin{array}{c}\text { Adaptive fuzzy } \\
\text { Control suspension }\end{array}$ & Reduction \\
\hline Body acceleration $\left[\mathrm{m} \cdot \mathrm{s}^{-2}\right]$ & $5.84 \times 10^{-1}$ & $4.27 \times 10^{-1}$ & $26.88 \%$ \\
Suspension displacement $[\mathrm{m}]$ & $3.82 \times 10^{-3}$ & $3.06 \times 10^{-3}$ & $19.90 \%$ \\
Tire deformation $[\mathrm{m}]$ & $5.19 \times 10^{-4}$ & $5.83 \times 10^{-4}$ & $-12.33 \%$ \\
\hline
\end{tabular}

\section{Conclusions}

1) On the basis of theoretical analysis and simulation tests, magneto-rheological damper based on the mixed mode was designed using obtained damping force model through data fitting. Results showed that it can meet the requirements of the aircraft tractor and also could be controlled through changing the size of the current.

2) Compared to the fuzzy control, adaptive fuzzy control is of higher precision, smaller overshoot, shorter adjustment time, and no oscillation. Adaptive fuzzy control can well improve vehicle ride comfort. And it is significantly better than the passive suspension.in both body acceleration and suspension travel.

3) The designed adaptive fuzzy control algorithm does not require domain-by-point conversion in the implementation process, which greatly reduces the amount of computation so as to enhance the real-time performance of control algorithm and reduce the requirements for hardware of control system and be easy to implement in engineering.

\section{Acknowledgements}

This work was financially supported by the National Natural Science Foundation of china (51275126) and Technology Development Program of Weihai (2012DXGJ13).

\section{Reference}

[1] Wang nengjian, Yang wanhui, Zhou lijie. Simulation Research on Rodless Aircraft tractor Pushing Job[J]. Mechanical Design. 2011, 28(9): 17-20.

[2] Zapateiro.Mauricio, Pozo.Francesc, Karimi.Hamid Reza, Luo. Ninqsu. Semiactive control methodologies for suspension control with magnetorheological dampers[J]. IEEE/ASME Transactions on Mechatronics. 2012, 17(2): 370-380.

[3] Kuznetsov.Alexey, Mammadov.Musa, Sultan.lbrahim, Hajil- arov.Eldar. Optimization of improved suspension system with inerter device of the quarter-car model in vibration analysis[J]. Archive of Applied Mechanics. 2011, 81(10): 1427-1437.

[4] Saifia.Dounia, Chadli.Mohammed, Labiod.Salim. Hळ control of multiple model subject to actuator saturation: Application to quarter-car suspension system[J]. Analog Integrated Circuits and Signal Processing. 2011, 69(1): 81-90.

[5] Kaldas.Mina M.S., Caliskan.Kemal, Henze.Roman, Kucu- kay.Ferit. Development of a Semi-Active Suspension Controller Using Adaptive-Fuzzy with Kalman Filter[J]. SAE International Journal of Materials and Manufacturing. 2011, 4(1): 505-515.

[6] Yu miao, Mao linzhang, Liao cangrong, Chen weimin. Vibration control for semi-active suspension based on magneto-rheological damper [J]. Sensors and Microsystems. 2007, 26(12): 34-40.

[7] Dong xiaomin, Yu miao, Liao cangmin, Chen weimin, Huang shanglian. Study on Magneto-Rheological Adaptive Fuzzy Semi-active Suspension Control [J]. China Journal of Highway. 2006, 19(2): 111-115.

[8] Fernando J.D’Amato, Daniel E.Viassolo. Fuzzy control for active suspensions[J]. Mechatronics. 
2000, 10(8): 897-920.

[9] Tzuu-Hseng S.Li, Chao-Lin Kuo, Nai Ren Guo. Design of an EP-based fuzzy sliding-mode control for a magnetic ball suspension system[J]. Chaos, Solitons\&Fractals. 2007, 33(5): 1523-1531. 